\title{
Application of Spectrum Data Management Based on Bayesian Network
}

\author{
Dai $\mathrm{Li}$ \\ Dept. of Computer Science \\ Yunyang Teachers' College, Shiyan 442000, China \\ lidai72@gmail.com
}

\begin{abstract}
In the spectral data management, under different regions and different time, spectral data is very big, resulting in the spectrum management would be difficult, this article based on Bayesian network of depth neural networks, aiming at the characteristics of spectrum management, studying the spectrum historical data, and according to the results to forecast spectrum occupancy, in order to achieve the spectrum management, the experimental results compared the spectrum of predictions and reality, and verified the effectiveness of the algorithm.
\end{abstract}

Keywords: Cognitive radio, spectrum management, data forecasting, Bayesian network

\section{Introduction}

Due to wireless communication has the characteristic that we can communicate possibly whenever and wherever, and its application is more and more widely, there are more and more communication equipment that applying wireless communication, the transmission of data is also growing exponentially; but the spectrum scarcity of natural resources, it needs to make full use of the spectrum. However, after investigation, due to previous spectrum management strategy is fixed, which is authorized to give specific users. However, the authorized users in the time dimension, spatial dimension and comprehensive dimensions, the spectrum is not fully utilized, so the cognitive radio technology is proposed in the late 90's of the last century, which is characterized by monitoring the implementation of the spectrum resources, and dynamically allocated spectrum to assign users at present moment and place really need to use this frequency. The proposed cognitive radio technology has been valued by academic, business applications, military communication aspects, and takes this as an opportunity; the related technology has been widely studied. In the applications, mainly in business communication, dynamic spectrum access to unauthorized users; in the military, the idle spectrum detection, communication in order to avoid the enemy for communication, and implying electronic warfare; in disaster recovery communications, which can use cognitive radio technology, organization can send and receive communication nodes, in order to form self-organization communication network, so as to realize the network reconstruction in short time.

In the process of implementing cognitive radio technology, the research began in the field of physical layer, i.e., spectrum sensing algorithm; however, the spectrum sensing technology is the foundation of cognitive radio technology, but cognitive radio is ultimately to complete spectrum allocation, spectrum sensing is investigated and studied the spectrum availability; how to carry out the distribution according to the spectrum sensing results, it is the core issue of cognitive radio technology; at the same time, because the cognition of spectrum is representative of the current time, 
however occupancy of the spectrum is dynamic, the sensing results cannot represent the occupation of the spectrum the next moment, so the spectrum allocation not only according to the occupancy of frequency spectrum of the current time, therefore, according to the historical data, predicting the future situation of the spectrum occupancy. However, due to the spectrum width is large, and it corresponds to different time and place, the record of the spectral information of data is very huge, in such big data condition, according to certain criteria, how to forecast the occupied spectrum accurately, according to the predicted spectrum occupancy, doing spectrum allocation intelligently, in order to achieve optimal configuration of the system resources, and improve the performance of the whole system.

Academic circles generally believed that: cross-layer design is the best way to solve issues related to cognitive radio. The main difference between cognitive radio and traditional radio transceiver is the operating parameters. In cognitive radio, there is no fixed work frequency, bandwidth and modulation mode, the transmission parameters depend on the following four processes: (a) spectrum sensing; (b) the spectrum handoff; (c) spectrum sharing; (d) spectrum management. Spectrum sensing includes monitoring neighbor users RF signal in the physical layer, determining the interference temperature and identifying the optimal transmission frequency band. The spectrum handoff is the secondary users in order to ens ure the quality of service performance, maintain seamless communication requirements in the transition band. Spectrum sharing and spectrum management includes the operation of physical access layer and network layer, such as scheduling, routing and QoS of application layer. Because the spectrum sensing, spectrum handoff, spectrum sharing and spectrum management is the base that CRN provides service, in accordance with the procedure, the spectrum sensing: in cognitive radio networks; according to the topology, in the radio environment, node is provided with sensing function, according to the detection algorithm the perception of the frequency spectrum, and the spectrum sensing report center node, or by using a distributed approach to management, the spectrum information of nodes sharing, for each node of intelligent spectrum access; the spectrum handoff: analyzing the spectrum information of each node, getting the idle spectrum, dynamic nodes related by the previous node switch to detect the idle spectrum; the spectrum handoff: handoff algorithm according to the different detection equipment performance and dynamic spectrum is different, the time is different, and the need for considering the transmitting end and the receiving end synchronous switching, facilitate the correct receiving end; spectrum sharing: providing fair spectrum allocation mechanism for the coexistence of multiple SU. In the design of the spectrum sharing mechanism can be mainly divided into three aspects: network structure based on the spectrum sharing mechanism, sharing the spectrum allocation based on behavior mechanism and sharing mechanism based on the spectrum access technology. The research of spectrum sharing mechanism mainly depends on two kinds of theories: the use of optimization and game theory to find the optimal spectrum sharing strategy; according to the dynamic spectrum management, spectrum with secondary users need to undertake business, according to the two aspects of the dynamic spectrum allocation scheme and the management of the business, so finally we can get a better spectrum allocation scheme. 


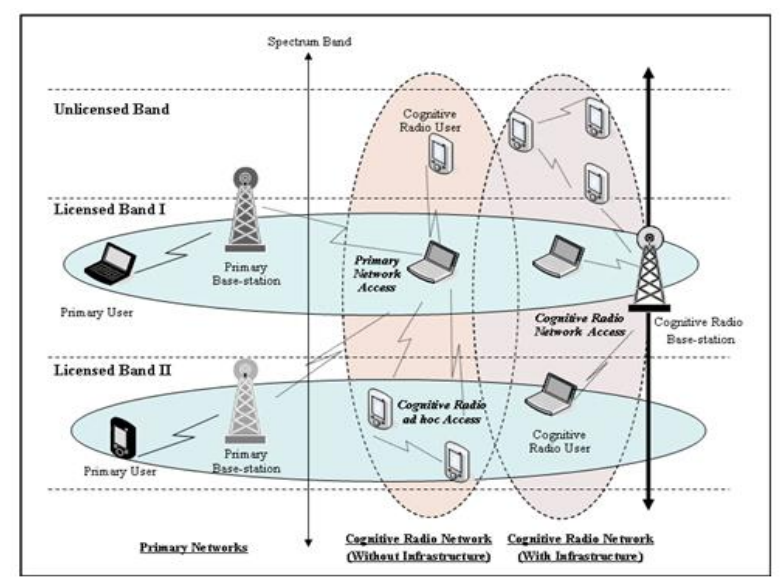

\section{Figure 1. Cognitive Radio Spectrum Sharing System Framework}

In the cognitive radio spectrum sharing system, the spectrum range is wide, we need to analyze and make reasonable judgment and distribution for the entire spectrum data, so it needs to establish a better data analysis structure. Bayesian network derived from the deep neural network of the field of artificial intelligence, the probability transfer function as a way to build connection level, it combined with probability theory and graph theory and mathematical theory, based on the combination of quantitative analysis and qualitative analysis, with the adaptive learning mode, building up the network architecture of Bayesian. This network architecture can be transferred between different network nodes according to the probability and conditional probability of transfer function, clear hierarchical indicates frequency spectrum of nodes. This article based on hierarchical Bayesian network to study cognitive radio spectrum sharing, according to the two part of the network structure and network parameters, getting the reasonable spectrum prediction and allocation algorithm. As shown in Figure 2, as the flow chart of the cognitive radio system.

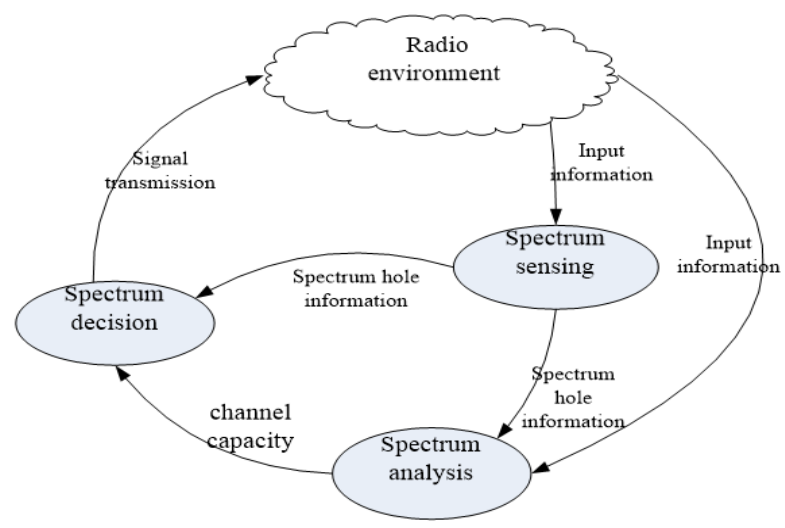

Figure 2. Cognitive Radio Schematic Diagram

\section{Related Works}

\subsection{The Research Status of Cognitive Radio}

Cognitive radio technology can be done sensing and transmission of the idle spectrum using a transceiver technology at the same time; in the spectrum sensing, according to the energy detection algorithm to sense the spectrum occupancy, but because the sensing process, some nodes are affected by path loss, shadow fading, 
the sensing accuracy would be affected, so the spectrum sensing by a single node to multiple nodes cooperative development, through multiple nodes of a spectrum, it can effectively avoid the sensing error because of shadow fading and path loss brought about However, due to multiple nodes sensing, how to fuse the multiple nodes sensing results, the study can be summarized as two fusion methods: hard decision and soft decision; these decisions directly affect the two sensing index, the probability of false alarm and false detection. Due to the introduction of multi antenna technology, application of multi antenna technology to sense the spectrum, it can effectively improve the detection accuracy of spectrum detection, so a large amount of literatures concentrated on multi antenna. When single spectrum sensing is studied, a lot of researches focused on the study of the spectrum of the integration mode. The topological structure of the fusion methods can be divided into centralized data fusion and non-centralized data. For centralized data fusion nodes, the detection result through the link to a data fusion center, the fusion center frequency according to the distribution of node demand. Therefore, the fusion center needs a strong signal processing ability, for the non-fusion center algorithm, through the mutual transmission of each node, it can get non-centralized spectrum sensing results [6]. Proposed a distributed spectrum detection of indoor conditions, so as to realize the non-fusion center algorithm; according to non-central data fusion algorithm [7], used the limited feedback manner, thereby reducing the amount of information transmission, it had certain innovation; according to on the theory of matrix [8], used the norm theory and LU decomposition, and the decomposition made the occupied channel states of primary users and secondary users are orthogonal to each other.

In order to obtain the most accurate wireless spectrum holes, making the center sensing node to produce a new handoff form, i.e., spectrum handoff. In the center of the cognitive network, when the channel condition detecting variation or to detect the primary users using the spectrum, secondary users need to switch frequency. In the spectrum handoff process, each layer of the protocol stack in the network must be changed in order to adapt to the characteristics of working frequency, and the delay of spectrum handoff and handover process of the protocol should be transparent. In order to realize the spectrum handoff function, the spectrum handoff management requirements must also deal with multilayer, these management protocols to support various types of business correspondence spectrum handoff. For example, in the spectrum handoff, TCP connections can be set into a wait state until after the restoration, the spectrum handoff. In addition, the spectrum handoff, transmission control protocol transmission parameters will change, so to learn new transmission parameters, and to ensure that the transition to the new parameters by the old parameters as rapidly as possible. For data transmission services (such as file transfer protocol), the spectrum handoff management protocol should perform storage mechanism to save the spectrum handoff to the transmission of data packets. For real-time traffic, the packet transmission delay is too large to be discarded, but not by the corresponding business use, so there is no need to store the spectrum handoff packet transmission. In the specific time and space, there may be several frequency bands for each user, according to the characteristic of the channel bandwidth and user service requirement; spectrum handoff algorithm should be able to determine the optimal switching frequency. When choosing the best available frequency band, the need for further design of handoff and connection management protocol, to reduce the delay and loss spectrum handoff process. If the user is currently used for communication bandwidth is the main user re occupied, the secondary user business must be transferred to other available frequency band transmission. However, it needs to consume some time off from work to the new band handoff the current use of the available frequency band. Therefore, it ensures 
that the smaller handoff algorithm for quality of service handoff process. In addition to detect the primary users to use radio frequency band, there are other reasons that the secondary users of spectrum handoff. For example, when a user moves, it probably because of changes in the available frequency band of spectrum handoff. Therefore, the spectrum handoff should also consider the handoff problem. In a word, in this dynamic, changing with time and space in the radio environment, in order to ensure the quality of service requirements, user must switch between different frequency bands.

\subsection{The Research Status of Bayesian Network}

When it comes to Bayesian networks, it originated the paper of mathematician Bayesian, in view of the prior probability distribution, according to the node topology structure and the independence of the other variables was studied. Bayesian networks have many similarities with human cognitive process; it can be divided into two aspects, qualitative and quantitative, the Bayesian network has knowledge representation from the qualitative and quantitative level. Bayesian network structure describes the relationship between variables, the qualitative relations; Conditional probability from the numerical describes the strength of the relationship between variables, it belongs to quantitative description [9]. Compared the human learning mechanism and the incremental learning of Bayesian networks, human learning new knowledge can be divided into two mechanisms which assimilation and compliance, Bayesian network learning process is similar. The inference of Bayesian network is very similar to the reasoning of the human brain, it is very suitable for advanced study of cognitive function.

Bayesian networks is first applied in the data mining, Bayesian networks can study has experienced three different stages. The first phase is studying the structure and theory system of Bayesian network, the second phase focuses on the study of learning algorithm of Bayesian network, including structure learning and parameter learning algorithm, and putting forward from learning algorithms; the third stage is in the field of practical problems are studied, especially for the Bayesian network model has been widely used in many practical problems, researching the most effective model. Bayesian network inference refers to the evidence given under the condition of the query Bayesian posterior information of some nodes in the network, the reasoning algorithm is divided into two major categories of precise reasoning algorithm and approximate reasoning algorithm. Precise reasoning algorithm mainly include: the message propagation algorithm, junction tree algorithm, symbol probability reasoning algorithm, arc deleting algorithm and derivative operator algorithm, etc., approximate reasoning algorithm mainly include: random sampling algorithm, the based on searching algorithm, reduction model algorithm and circulation message propagation algorithm and so on. Bayesian network learning two aspects including structure learning and parameter learning, learning is simple specification, Bayesian network learning research mainly focused on the study of Bayesian network structure learning algorithm. The structure of the Bayesian network learning process is combined with the prior information of the including expert knowledge, to find the best fitting with sample data set network structure. Since the $1990 \mathrm{~s}$, researchers from different angles of Bayesian network learning problems are studied, they proposed many classic Bayesian network learning algorithm, these algorithms can be roughly divided into three broad categories: based on the score of search method, based on the analysis of the dependence and blended learning method. Which is based on the analysis of the dependence is also known as the method based on independence test, the method to use chi-square test or mutual information in information theory to judge the independence between variables and conditional independence, and describe dependencies between 
variables and independent relations on the basis of Bayesian network. Based on the analysis of the dependence of the accuracy and the learning efficiency depends on the learning process of the order number and the number of conditional independence test, and in the process of learning to be the number of conditional independence test and order depends on the complexity of the network structure, so the algorithm is suitable for the sparse Bayesian network structure learning. This article is based on sparse Bayesian network structure, studying the spectrum allocation, handoff and prediction of spectrum data.

\section{Proposed Scheme}

In this paper, according to the structure of Bayesian network and the spectrum sensing data during network nodes, the transition probability and relationship, occupancy of the spectral data for data mining, data prediction. We need to determine the relevant parameters of Bayesian network; at the same time, according to the forecast data, and the Bayesian network structure optimization, the management of the entire data, the spectrum management algorithm is proposed. Therefore, the proposed scheme is divided into two parts, the first part is the data forecasting based on Bayesian network; the second part is the spectrum management based on Bayesian network.

\subsection{Data Forecasting Based on Bayesian Network}

In the data forecasting, it is difficult to find a precise definition that a clear set of spectrum has been occupied and non-occupied. Thus, it needs to use the concepts of fuzzy sets to establish the related concepts of Bayesian networks. If setting $\mathrm{x}$ is the set of all possible values of object $x$, so we call $\mathrm{x}$ is the domain of discourse of $x$.And if the set of $\mathrm{X}$ is $B=\left\{\left(x, \theta_{B}(x) \mid x \in X\right)\right\}$, where, $\theta_{B}(x)$ represents the degree of $x$ belongs to $B$, its values between 0 and 1 , the closer to 0 , the more do not belong to $B$, the closer to 1 , the more belong to $B$. According to the value of $B$, we can measure the degree of closeness to $x$. For the special case of Bayesian networks under fuzzy set, which can describe the nonlinear system, and the system has in the dimension of time, so it can be suitable for the data forecasting. According to the basic structure of Bayesian network, we can build the fuzzy Bayesian network. As shown in Figure 3, the initial Bayesian network and transfer network. We set $\mathbf{X}=\left[\mathrm{X}^{1}, \mathrm{X}^{2}, \cdots, \mathrm{X}^{n}\right]$ is the random variable set, where $\mathrm{X}_{t}^{i}$ is the variable at time $t$. As can be seen from the Figure, the transition probability can be described by (1)

$$
\mathrm{P}\left(\mathrm{X}_{t}^{i} \rightarrow \mathrm{X}_{t}^{i+1}\right)=\operatorname{Pr}\left(\mathrm{X}_{t}^{i+1} \mid \mathrm{X}_{t}^{i}\right)
$$

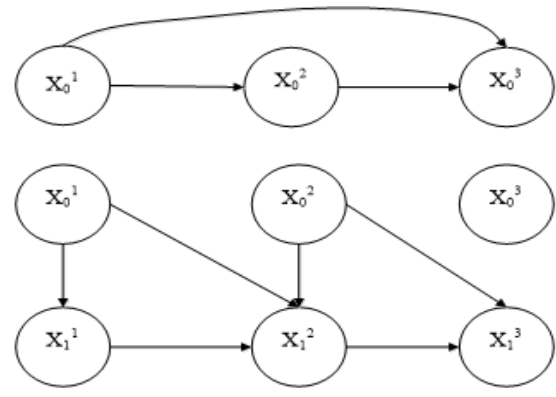

Figure 3. Bayesian Network Structure

The joint distribution probability is: 


$$
\operatorname{Pr}\left(X_{i}\right)=\prod_{i=1}^{n} \operatorname{Pr}\left(X_{t}^{i+1} \mid X_{t}^{i}\right)
$$

So we can get the probability distribution of a set of $\mathrm{K}$ in the Bayesian network:

$$
\operatorname{Pr}\left(X_{i}\right)=\operatorname{Pr}\left(X_{0}\right) \prod_{i=1}^{n} \operatorname{Pr}\left(X_{t}^{i+1} \mid X_{t}^{i}\right)
$$

For transition probability of Markova chain, because of the data set is big, that all the transfer probability distribution follow Law of Great Numbers, i.e., Gaussian distribution, that is

$$
\operatorname{Pr}\left(\mathrm{X}_{t}^{i+1} \mid \mathrm{X}_{t}^{i}\right) \square N\left(\mu_{t}, \sigma_{t}^{2}\right)
$$

According to this reasoning, the topology matrix composed by each node is obtained:

$$
S_{X}=\left\{\begin{array}{cccc}
S_{11} & S_{12} & \cdots & S_{1 k} \\
S_{21} & S_{22} & \cdots & S_{2 k} \\
\cdots & \cdots & \cdots & \cdots \\
S_{m 1} & S_{m 2} & \cdots & S_{m k}
\end{array}\right\}
$$

Based on this algorithm, we can get data forecasting process

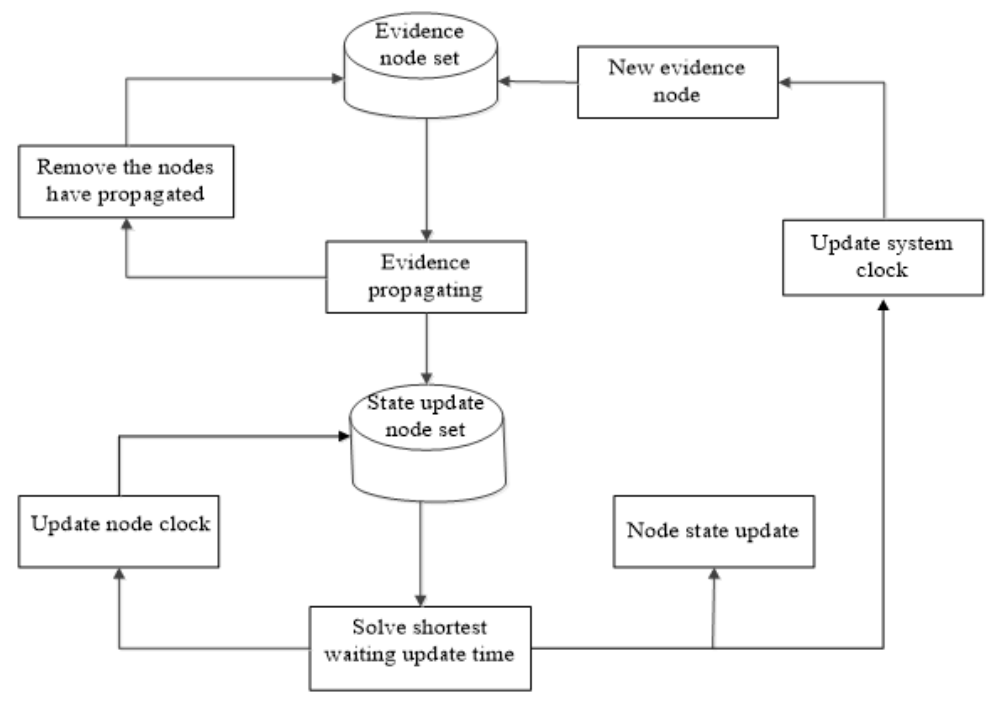

Figure 4. Bayesian Network Data Forecasting Process

\subsection{Spectrum Management Based on Bayesian Network}

To the occupancy for dynamic radio spectrum, according to the previous spectrum occupancy data, it can be data forecasting as shown in Section 3.1, to predict the spectrum occupancy. According to the forecast data, the spectrum should be re allocation and spectrum structure should be reconstruction. In the cognitive radio system, how to make use of cognitive search function, intelligent adjustment and re configuration, modulation, channel coding, transmission power, bandwidth and other parameters. But in the environment parameters, including the mobile node location information, the spectrum information, interference information and the moving speed, etc., we can use $M=\left\{M_{1}, M_{2}, \cdots, M_{n}\right\}$, and $C=\left\{c_{1}, c_{2}, \cdots, c_{n}\right\}$ represents 
configure transmission parameters. Considering the user's business needs, resource allocation and the corresponding requirements of business, such as time, power and frequency and so on, they are represented by $S=\left\{s_{1}, s_{2}, \cdots, s_{n}\right\}$.The end-to-end performance also expressed in matrix $Q=\left\{q_{1}, q_{2}, \cdots, q_{n}\right\}$. Cognitive learning as the core of cognitive engine based on BN, Mainly by the Bayesian network structure learning algorithm and parameter learning algorithm to realize, and it used to obtain knowledge of the guidance, cognitive reasoning decision-making configuration parameters.

According to spectrum management and the related data characteristics of resources allocation, creating a sample database, according to the self-learning method to determine the sample database, a cognitive model of structure calculation, according to the mutual relation between the communications, establishing a correlation network model, the network model of correlation model is

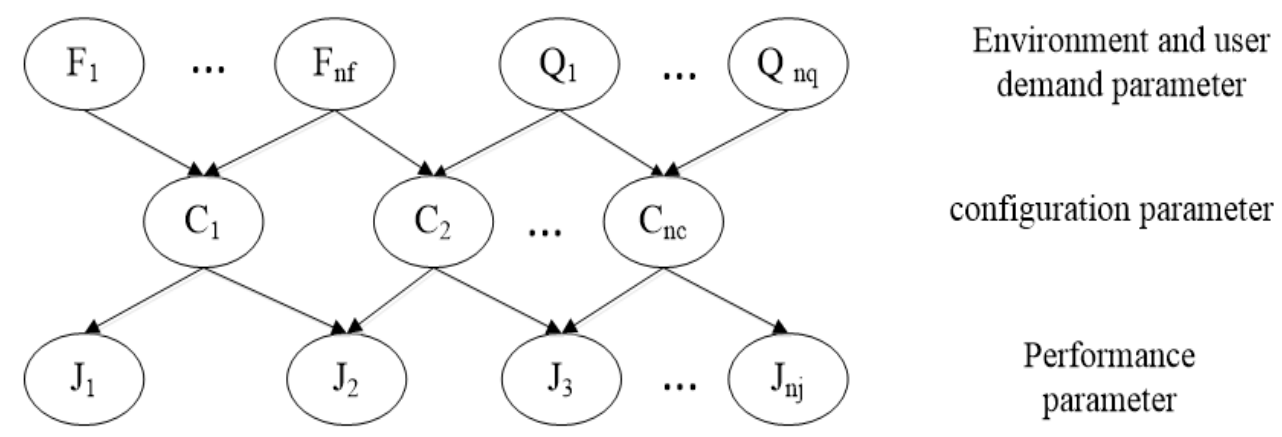

Figure 5. Bayesian Model Structure

For the optimization function of maximization, it needs to use the posterior probability of correlative node, the conversion using Bayesian probability, the prior probability is transformed into the posterior probability, as shown in(6)

$$
P\left(X_{i} \mid X_{j}\right)=\frac{P\left(X_{j} \mid X_{i}\right) P\left(X_{i}\right)}{P\left(X_{j}\right)}
$$

Traversing all the communication parameters nodes in turn, adding nodes and delete nodes for network, adding or deleting the directed edges, and then according to the evaluation function to evaluate the network structure, the structure model of the highest score as a mobile wireless network in the Bayesian network structure. According to the characteristics of cognitive radio spectrum management, the final optimized function is

$$
\max [c(\mathrm{~S}, \mathrm{M}, \mathrm{Q})]=P(S) \max [M Q]
$$

And after determine the optimized function, related parameters of network structure need to be determined, using maximum likelihood function method, and the related parameters of network architecture are obtained by derivation finally. In the decision-making process, the process can be divided into the following steps:

Step 1, according to the node in the model to connect one to one, according to the relationship between the topology, removing duplicate edges;

Step 2, based on the principle of triangulation subdivision, completing the triangle topology structure, according to the segmentation of triangulation, spectrum resources and related resources would be allocated; 
Step 3, according to the specific distribution of triangular subdivision, determining the transmission scheme, according to the transmission scheme, estimating the related performance of the point-to-point, such as capacity, bit error rate, etc.,

Step 4, according to the performance of the point-to-point, estimating the overall performance of the whole system;

Step 5, return to Step 2 until the system overall performance become convergence.

\section{Experiment Results and Analysis}

In order to evaluate the performance of $\mathrm{BN}$ cognitive engine, in this paper, the mobile wireless network communication model of OMNET simulation software was used to establish the communication events, obtaining the desired cognitive engine data, and completing the configuration simulation experiment. The mobile wireless network shown in Figure 4, $t x$ as the sending node, $r x$ as the receiving node, jam as the interference node, sending node and interference node along the path shown in the Figure, and the position of receiving node is unchangeable. In order to reflect the dynamic and variable of network environment, the interference node from far to near to the receiving node and then move further away from the receiving node, and sending the interference signal interference the data transmission between sending node and receiving node. The parameter settings in detail as shown in Table 1

Table 1. Cognitive Radio Parameters Configuration

\begin{tabular}{cc}
\hline Parameter type & Parameter value \\
\hline Channel fading & Free space model \\
Traffic type & Voice,Video,Ftp,Email \\
Power & $0.001,01005,0.01,0.05 / \mathrm{W}$ \\
Modulation system & BPSK,GMSK,16QAM \\
Bandwidth & $10,100 / \mathrm{kHz}$ \\
Sending data rate & $1024,10240 / \mathrm{bps}$ \\
\hline
\end{tabular}

The simulation path is shown in Figure 6, according to the number of business model and the scheduling of resources and the data flow, the core algorithm of data forecasting, according to the data forecasting, the average delay of end-to-end can be obtained, this delay can be determined according to difference of the interval simulation environment, it needs to be set separately.

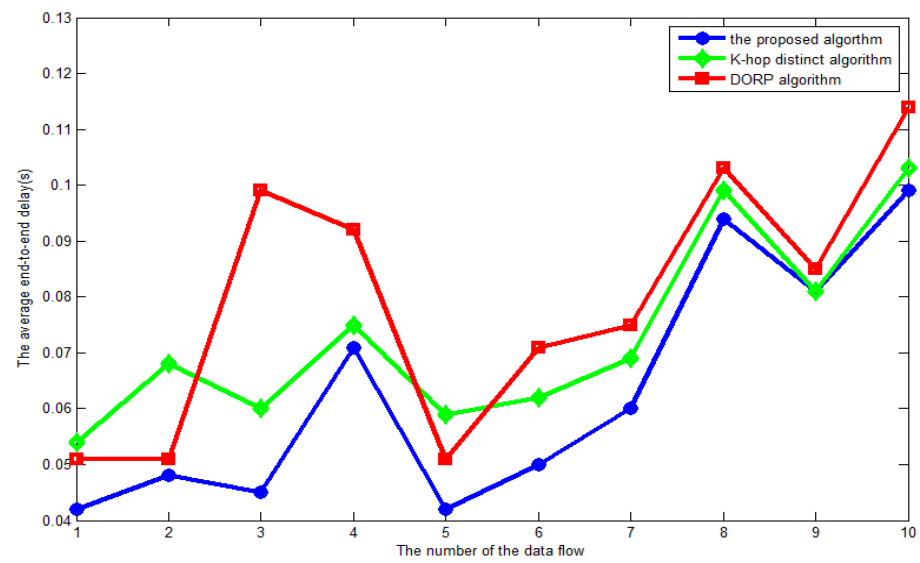

Figure 6. Average Energy Consumption Comparison 
Figure 6 shows that the improved scheme is proposed in this paper, the advantage in the end to end, according to the different topology structure and the execution time, the transmission delay has difference. And with the data arrival rate is growing, the advantage is more obvious. As can be seen, because the data arrival rate distribution of the tourism business is broad, i.e., with the passage of time, there will be unexpected data arrives, but the energy consumption is the lowest in the whole data arrival rate, the scheme has very strong robustness. Thereby, it saved time of end to end.

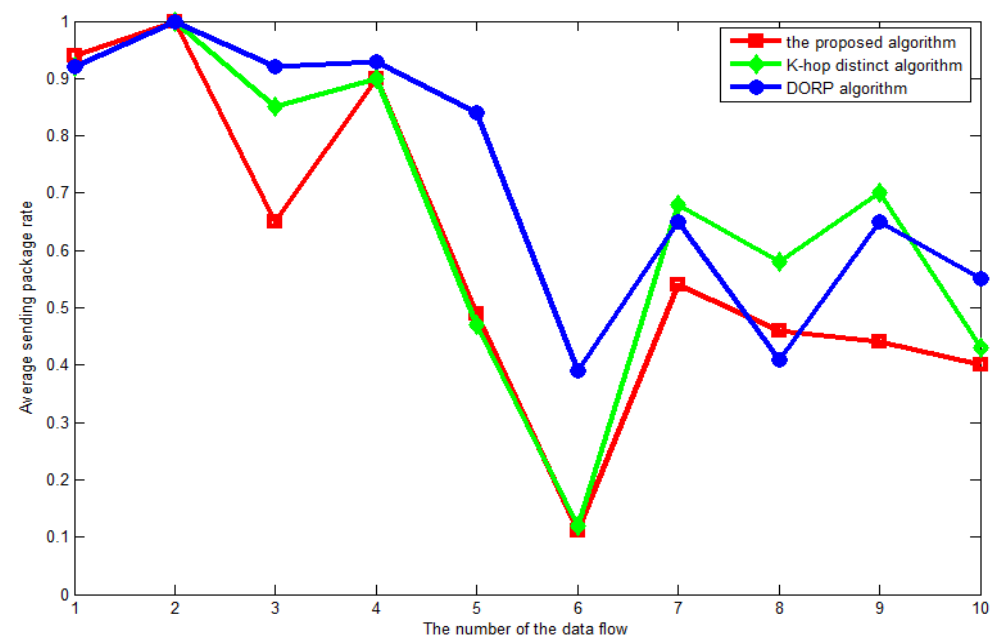

Figure 7. Resource Utilization Comparison

From the reliable transmission of the data, we can see in Figure 7, the average packet loss probability of the proposed scheme significantly lower than the other schemes when the data flow has increased, the proposed scheme is effective in the reliable transmission; among them, spectrum management using Bayesian structure, spectrum allocation and related transmission mode adjustment is more effective.

\section{Conclusion}

In this paper, according to the spectrum cognitive and management of cognitive radio, spectrum cognitive furcating, the spectrum management and related transmission mode management were designed, in the design, using the Bayesian network structure from depth of neural networks, it can effective learning related data, and carry on forecasting and distributing of the relevant data, the simulation results have verify the validity of the scheme.

\section{References}

[1] Z. Han, R. Zheng and H. V. Poor, "Repeated auctions with Bayesian nonparametric learning for spectrum access in cognitive radio networks", Wireless Communications, IEEE Transactions on, vol. 10, no. 3, (2011), pp. 890-900.

[2] B. Wang, Y. Wu and K. J. R. Liu, "Game theory for cognitive radio networks: An overview", Computer networks, vol. 54, no. 14, (2010), pp. 2537-2561.

[3] Y. Gai, B. Krishnamachari and R. Jain, "Learning multiuser channel allocations in cognitive radio networks: A combinatorial multi-armed bandit formulation", New Frontiers in Dynamic Spectrum, IEEE Symposium on IEEE, (2010), pp. 1-9.

[4] W. Wang, H. Li and Y. L. Sun, “Attack-proof collaborative spectrum sensing in cognitive radio networks", Information Sciences and Systems, CISS, 43rd Annual Conference on IEEE, (2009), pp. 130-134. 
[5] A. S. Rawat, P. Anand and H. Chen, "Collaborative spectrum sensing in the presence of byzantine attacks in cognitive radio networks", Signal Processing, IEEE Transactions on, vol. 59, no. 2, (2011), pp. 774-786.

[6] P. Balamuralidhar and R. Prasad, "A context driven architecture for cognitive radio nodes", Wireless Personal Communications, vol. 45, no. 3, (2008), pp. 423-434.

[7] X. Xing, T. Jing and Y. Huo, "Channel quality prediction based on Bayesian inference in cognitive radio networks", INFOCOM, Proceedings IEEE, (2013), pp. 1465-1473.

[8] X. Xing, T. Jing and W. Cheng, "Spectrum prediction in cognitive radio networks", Wireless Communications, IEEE, vol. 20, no. 2, (2013), pp. 90-96.

[9] W. Wang, H. Li and Y. Sun, "Securing collaborative spectrum sensing against untrustworthy secondary users in cognitive radio networks", EURASIP Journal on Advances in Signal Processing, (2010), pp. 4.

\section{Author}

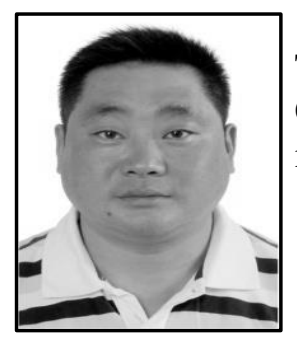

Dai Li, born in 1972, an associate professor of Yunyang Teachers' College in China. He got a Master's degree in Computer Science. He is mainly researching on computer network, computer science education. 
International Journal of $u-$ and $e-$ Service, Science and Technology Vol.8, No. 7 (2015) 